\title{
MULTIPLE SKIN AND BRAIN ABSCESS CAUSED BY SALMONELLA TYPHI, FIRST REPORTED CASE IN BANGLADESH
}

\author{
MAHMUDUR RAHMAN SIDDIQUI, QUAZI TARIKUL ISLAM, YOUSUF UR RAHMAN, \\ TAMANNA YASMIN SHENA
}

\begin{abstract}
:
Focal Brain and skin abscesses due to Salmonella are rarely reported. They tend to occur in patients who are immunosuppressed and in those with other predisposing factors like; sickle cell disease. We presented a case of a 28-year-old female of multiple skin and brain abscesses caused by Salmonella Typhi with no predisposing factor. After starting treatment according to culture and sensitivity report, she completely recovered without any residual disability.
\end{abstract}

Key words: Salmonella Typhi, brain abscess, skin abscess

Received: 4 January 2015

Accepted: 3 March 2015

\section{Introduction:}

Salmonella enterica causes a variety of human infections in the tropic. In India, the most common serotypes causing human infections are Salmonella Typhi (73\%) and Salmonella Paratyphi A (24\%) among typhoidal serovars, and Salmonella Worthington (28.2\%) and Salmonella Typhimurium (22.5\%) among non-typhoidal serotype. ${ }^{1}$ Salmonella typhi infection usually presents as enteric fever and gastroenteritis. Some Salmonellae may cause septicemic disease with focal suppurative lesions, such as osteomyelitis, deep abscesses, endocarditis, pneumonia and meningitis. ${ }^{2}$ Associated gastroenteritis may or may not be present. $^{2}$ To the best of our knowledge, this case report is the first description of a Salmonella typhi infection producing both skin and brain abscess in Bangladesh.

\section{Case Report:}

A 28-year-old female was admitted to Dhaka Medical College Hospital, with complaints of multiple painful discharging abscesses in the skin of left leg and scalp for 3 months, progressive headache for 2 months. She was treated with various antibiotics (Flucloxacillin, Cefradine), local dressing for her skin abscess but there was no significant improvement. The entire abscess became non healing ulcer after the drainage of the pus. For last 2 months she felt headache which was constant dull aching in nature, first localized to the right side then gradually involved the whole head. Headache aggravated at early morning by bending forwards, sometimes associated with nausea and vomiting. During this period of illness she developed occasional high grade intermittent fever. For last 2 weeks she developed gradual slurring of speech followed by motor aphasia. It was associated with deviation of mouth towards left side. She was non diabetic. Clinical examination revealed multiple non healing skin ulcers in left leg and scalp. She had motor aphasia, right sided upper motor type of $7^{\text {th }}$ cranial nerve palsy with right sided extensor planter reflex. Fundoscopic examination revealed bilateral papilloedema.

Laboratory investigations revealed haemoglobin 10 $\mathrm{g} / \mathrm{dl}$, white blood cells count of $18000 / \mathrm{mm} 3$ with neutrophil $80 \%$, peripheral blood film showed neutrophilic leucocytosis. Urine routine examination, random blood sugar, serum creatinine and chest $\mathrm{x}$ ray revealed normal findings. Pus from different wound for culture \& sensitivity revealed Salmonella Typhi. MRI of the brain revealed one big ring enhancing lesion with peri-lesional oedema at the left parietal region with two small enhancing satellite lesions, suggestive of multiple left parietal abscess of the brain.

According to culture sensitivity report we started treatment with ceftriaxone $4 \mathrm{gm}$ daily for 2 weeks and dexamithasone $5 \mathrm{mg} 8$ hourly for 5 days with supportive care. On the $3^{\text {rd }}$ day of treatment her headache improves significantly. From the $6^{\text {th }}$ day of

1. Assistant Professor of Medicine, Anwer Khan Modern Medical College.

2. Professor of Medicine, Popular Medical College.

3. Registrar, Dhaka Medical College.

4. Postgraduate Resident, Dhaka Medical College Hospital.

Address of Correspondence: Dr. Mahmudur Rahman Siddiqui, Assistant Professor of Medicine, Anwer Khan Modern Medical College. E-mail: dr.mahmud99@yahoo.com.

Bangladesh J Medicine 2015; 26 : 83-85 
treatment, she started talking with some difficulties and all the ulcer began to heal. After 2 weeks she started to talk with out any difficulties. Her headache completely subsided and most of the skin ulcers were near to heal, but still she had right sided $7^{\text {th }}$ cranial nerve palsy with extensor planter reflex. She was discharged with tablet cefixime $200 \mathrm{mg}$ twice daily for another 2 weeks. After 4 weeks she came to us on follow up visit and revealed she had no complaints, all the skin ulcers were completely healed, having no dysarthria, no $7^{\text {th }}$ cranial nerve palsy and no papilloedema. Follow up MRI of the brain after 2 months was completely normal.

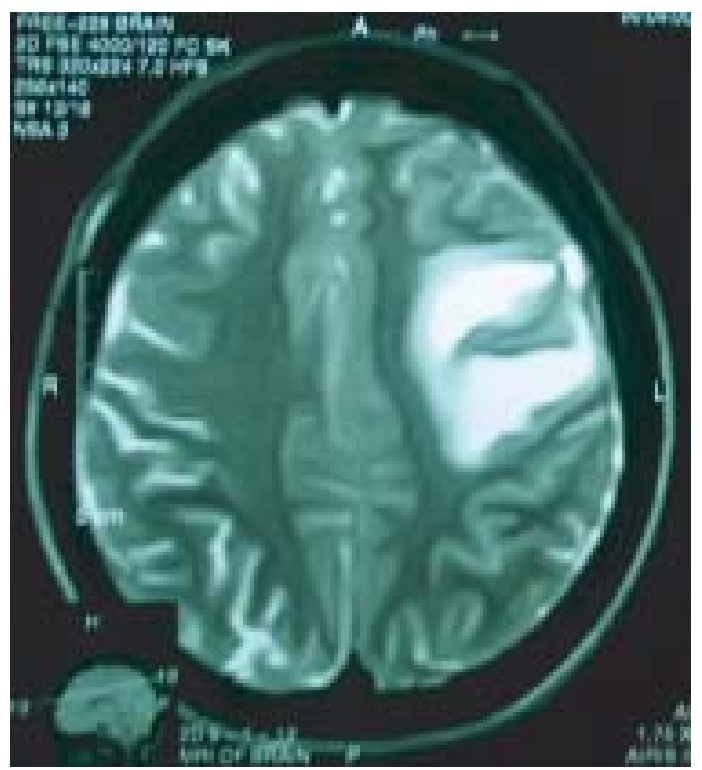

Fig.-1: T2 weighted axial MRI of the brain showing hyper intense shadow in left parietal region.

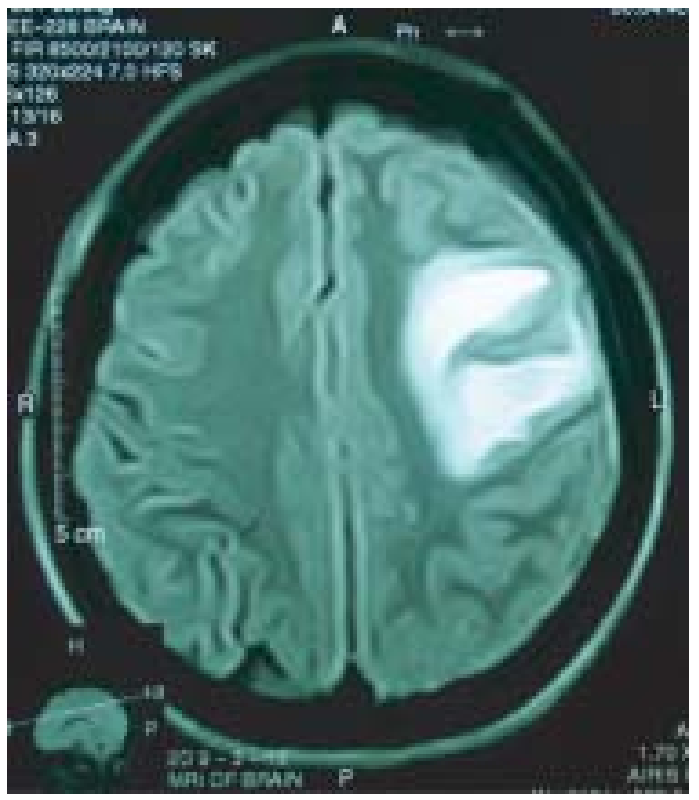

Fig.-2: Flair weighted axial MRI of the brain showing hyper intense shadow in left parietal region.

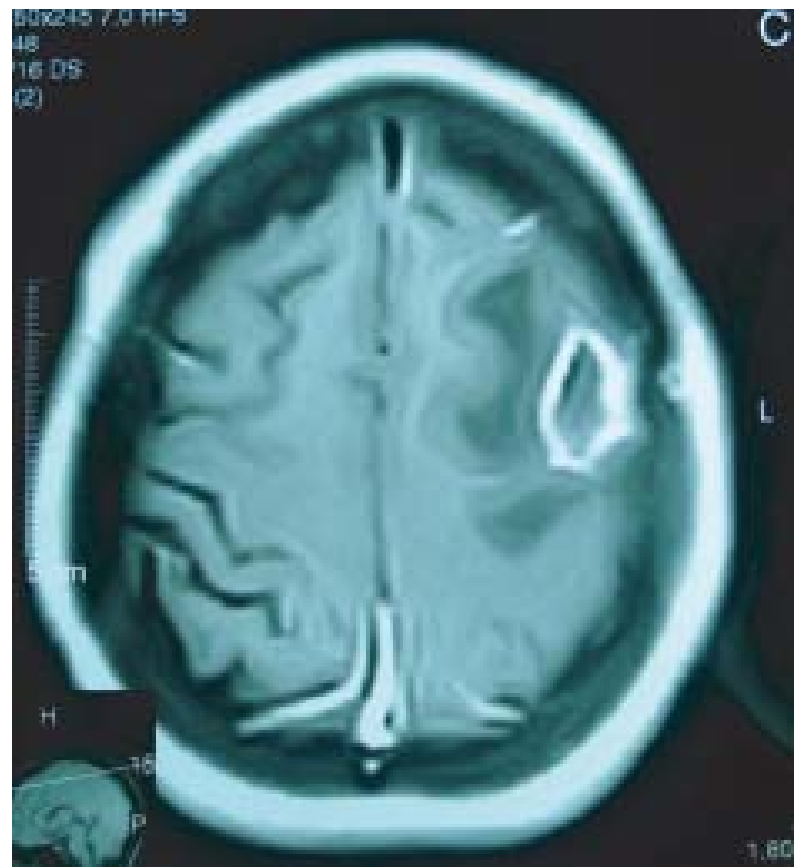

Fig.-3: Post contrast MRI shows a big ring shadow with perilesional oedema in the left parietal region.

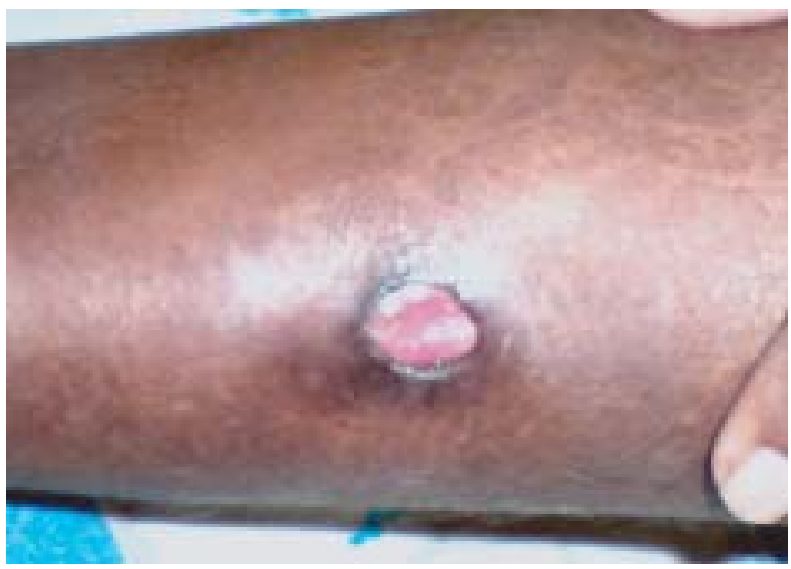

Fig.-4: Ulcer in the shin of the left leg after drainage of the abscess.

\section{Discussion}

Salmonella brain abscesses are a rare condition complicating salmonellosis, even in endemic areas. To the best of our knowledge, Salmonellae typhi have never been reported to cause multiple skin and brain abscess together. Salmonella has a tendency to infect diseased tissue, and a variety of predisposing factors have been described for brain abscess. ${ }^{3}$ These include patients with primary or metastatic brain tumors, subarachnoid hemorrhage, corticosteroid use, diabetes mellitus, immunoglobulin deficiencies, HIV infection, and meningitis. Meningitis is the most important predisposing factor to focal intracranial infection by Salmonella. ${ }^{3,4}$ Brain abscesses due to 
Salmonella spp, including S. enteritidis, have previously been reported and are usually assumed following bacteremia in the presence of some compromise of the reticulo-endothelial system (RES) immunity. Beta-thalassemia and sickle cell disease (SCD) are known to lead to RES compromise. ${ }^{5}$

Mahapatra et al 6 in a review of cases of focal intracranial Salmonella infections in the world literature between 1884 and 2001, reviewed 80 reported cases of focal intracranial Salmonella infections, of which 22 were brain abscesses, 40 subdural empyemas and 18 were combinations of abscesses, empyemas, and epidural abscesses. ${ }^{3,5,6}$ According to them, brain abscesses were common in adults and subdural empyemas more frequent in children. In general, their prognosis is poor with a mortality rate as high as $21-60 \%$ described in some patient series and a significant number of survivors developed permanent neurological sequelae. 5,6,7 salmonella localization to the skin presenting as cutaneous ulceration is regarded as a rare event.

Management of Salmonella brain abscesses requires a combination of antimicrobial agents, surgical drainage/excision as appropriate. ${ }^{3,5}$ Third-generation cephalosporins are the most sensitive agents to treat Salmonella CNS infections because they achieve high brain tissue concentrations. The recommended duration of antibiotic treatment is 4 to 6 weeks or longer, with clinical and radiological monitoring. ${ }^{3,5}$

Conflict of interest: we have no conflict of interest.

\section{References:}

1. Kumar Y, Sharma A, Sehgal R, et al. Distribution trends of Salmonella serovars in India (2001-2005). Trans $R$ Soc Trop Med Hyg 2009;103:390-94.

2. Desikan P, Kumar Y, Pande HK, et al. Isolated ulcerative skin lesion caused by Salmonella Weltevreden. J Infect Dev Ctries 2009;3(7):569-71.

3. Blázquez D, Muñoz M, Gil C, et al. Brain abscess and epidural empyema caused by Salmonella enteritidis in a child: successful treatment with ciprofloxacin: a case report. Cases Journal 2009, 2:7131 doi: 10.1186/1757-1626-2-7131.

4. Rodriguez RE, Valero V, Watanakunakorn C. Salmonella focal intracranial infections: review of the world literature (1884-1984) and report of an unusual case. Rev Infect Dis 1986, 8:31-41.

5. Kuruvath S, Basu S, Elwitigala JP, et al. Salmonella enteritidis brain abscess in a sickle cell disease patient: case report and review of the literature. International Journal of Infectious Diseases 2008; 12: 298-302.

6. Mahapatra AK, Pawar SJ, Sharma RR. Intracranial Salmonella infections: meningitis, subdural collections and brain abscess. A series of six surgically managed cases with follow-up results. Pediatr Neurosurg 2002, 36:8-13.

7. Hanel R, Araújo J, Antoniuk A, et al. Multiple brain abscesses caused by Salmonella typhi: case report. Surg Neurol 2000, 53:86-90.

8. Marzano AV, Mercogliano M, Borghi A, et al. Cutaneous infection caused by Salmonella typhi. $J$ Eur Acad Dermatol Venereol 2003 Sep; 17(5):575-7. 